\title{
Photoinduced quantum spin and valley Hall effects, and orbital magnetization in monolayer $\mathrm{MoS}_{2}$
}

\author{
M. Tahir, ${ }^{*}$ A. Manchon, and U. Schwingenschlögl ${ }^{\dagger}$ \\ PSE Division, KAUST, Thuwal 23955-6900, Kingdom of Saudi Arabia
}

(Received 19 April 2014; revised manuscript received 10 September 2014; published 22 September 2014)

\begin{abstract}
We theoretically demonstrate that $100 \%$ valley-polarized transport in monolayers of $\mathrm{MoS}_{2}$ and other group-VI dichalcogenides can be obtained using off-resonant circularly polarized light. By tuning the intensity of the off-resonant light the intrinsic band gap in one valley is reduced, while it is enhanced in the other valley, enabling single valley quantum transport. As a consequence, we predict (i) enhancement of the longitudinal electrical conductivity, accompanied by an increase in the spin polarization of the flowing electrons, (ii) enhancement of the intrinsic spin Hall effect, together with a reduction of the intrinsic valley Hall effect, and (iii) enhancement of the orbital magnetic moment and orbital magnetization. These mechanisms provide appealing opportunities to the design of nanoelectronics based on dichalcogenides.
\end{abstract}

DOI: 10.1103/PhysRevB.90.125438

PACS number(s): 73.63.-b, 72.20.Ht, 73.43.-f

Monolayers of the transition metal dichalcogenides $M X_{2}$ ( $M=\mathrm{Mo}, \mathrm{W} ; X=\mathrm{S}, \mathrm{Se}$ ) are emerging as promising materials for a wide variety of applications in nanoelectronics, due to their exceptional band structures [1]. In particular, the exfoliation of $\mathrm{MoS}_{2}$ monolayers has attracted significant interest since the realization of field-effect transistors with high on-off ratio [2]. Monolayers of $M X_{2}$ can be regarded as semiconductor analogs of graphene $[3,4]$, resulting in similar phenomena such as spin and valley Hall effects [5,6]. Indeed, $\mathrm{MoS}_{2}$ has a honeycomb lattice with an intrinsic direct band gap of 1 to $2 \mathrm{eV}$, which is in the visible range. The band edge is located at the energy degenerate valleys (corners of the hexagonal Brillouin zone) [7,8].

Thanks to its direct band gap, $\mathrm{MoS}_{2}$ is suitable for optical manipulations and opens access to many optoelectronic applications [7-9]. It has been predicted that both valley polarization and valley coherence can be achieved by optical pumping with circularly and linearly polarized light $[5,6,10]$. Experimental realizations have been reported for $\mathrm{MoS}_{2}$ and $\mathrm{WSe}_{2}$ [11-14], suggesting that monolayers of $M X_{2}$ could be used for integrated valleytronic devices. Experiments have shown $30 \%$ to $50 \%$ valley polarization with circularly polarized light in the resonance regime [10-12]. Recent works on the optoelectronic properties of $\mathrm{MoS}_{2}$ indicate that the photoresponse of externally biased phototransistors is driven by conductivity alteration upon illumination [15-17]. A photovoltaic effect has been reported for $\mathrm{MoS}_{2}$ devices in contact with metallic electrodes that generate large Schottky barriers [18,19]. In addition, ultrasensitive phototransistors with improved mobility have been demonstrated in Ref. [20]. These devices show a photoresponsitivity in the 400 to $680 \mathrm{~nm}$ range with a maximum of $880 \mathrm{~A} / \mathrm{W}$ at a wavelength of $561 \mathrm{~nm}$.

In contrast with the on-resonant optical induction used till now, we propose in this paper a scheme to employ off-resonant light to influence the band structure and corresponding transport properties of $M X_{2}$ monolayers, enabling $100 \%$ valley polarization. An important motivation is the development of experimental probes [21] that make it possible to access nonequilibrium effects, where time-periodic perturbation due

\footnotetext{
*m.tahir06@alumni.imperial.ac.uk

†udo.schwingenschlogl@kaust.edu.sa
}

to circularly polarized light represents a rich and versatile resource for creating a band gap [22]. An analog has been realized experimentally in a photonic system $[23,24]$. We show that the band structure of $\mathrm{MoS}_{2}$ is strongly modified by the off-resonant light, resulting in a valley-dependent tuning of the band gap, which is not achievable by on-resonant light. We demonstrate analytically that this valley-dependent band gap results in the emergence of a quantum spin Hall effect, the reduction of the quantum valley Hall effect, and enhancement of the orbital magnetic moment and orbital magnetization.

Off-resonant light cannot generate real photon absorption/emission due to energy conservation, whereas it can affect the electron system by second order virtual photon processes (a photon is first absorbed/emitted and then emitted/absorbed). When averaged over time, these processes result in an effective static alteration of the band gap of the system. Therefore, using circularly polarized light, it is possible to distinctively tune the band gap at the $K$ and $K^{\prime}$ valleys. Gap opening by off-resonant light has been predicted for graphene and the surface states of topological insulators [22] as well as for silicene [25], and has been confirmed experimentally [21]. These studies show that off-resonant light enables quantum phase transitions in two-dimensional systems.

The charge carriers in $\mathrm{MoS}_{2}$ obey a two-dimensional Diraclike Hamiltonian [5,6] with large intrinsic direct band gap and strong spin-orbit coupling (SOC). We model $\mathrm{MoS}_{2}$ by an effective Hamiltonian in the $x y$ plane in the presence of circularly polarized light,

$$
\hat{H}^{\eta, s}(t)=v\left[\eta \hat{\sigma}_{x} \hat{\Pi}_{x}(t)+\hat{\sigma}_{y} \hat{\Pi}_{y}(t)\right]+\Delta \hat{\sigma}_{z}-s \eta \lambda \hat{\sigma}_{z}+s \eta \lambda .
$$

Here $\eta= \pm 1$ represents the valleys $K$ and $K^{\prime}$, respectively, $\Delta$ is the mass term that breaks the inversion symmetry, $\left(\hat{\sigma}_{x}\right.$, $\hat{\sigma}_{y}, \hat{\sigma}_{z}$ ) is the vector of Pauli matrices (applies to both the valence and conduction bands), $\lambda$ is the SOC energy, $s= \pm 1$ represents the up and down spins, respectively, and $v$ denotes the Fermi velocity of the Dirac fermions. In our notation, the spin-quantization axis is chosen along the $z$ direction. We use the gauge in the two-dimensional canonical momentum $\hat{\mathbf{\Pi}}(t)=\hat{\mathbf{P}}-e \mathbf{A}(t)$, with the vector potential

$$
\mathbf{A}(t)=( \pm A \sin \Omega t, A \cos \Omega t),
$$


where $\Omega$ is the frequency of the light and $A=E_{0} / \Omega$ with $E_{0}$ being the amplitude of the electric field $\mathbf{E}(t)=\partial \mathbf{A}(t) / \partial t$. The gauge potential satisfies time periodicity $A(t+T)=A(t)$ with $T=2 \pi / \Omega$. The plus/minus sign refers to right/left circular polarization. As long as the photon energy is much larger than the kinetic energy of the electrons, $\hat{H}^{\eta, s}(t)$ can be reduced to an effective static (time-independent) Hamiltonian $\hat{H}_{\text {eff }}^{\eta, s}$ using Floquet theory [22,23]. This method gives results in excellent agreement with experiments $[21,23,24]$. The effective Hamiltonian $\hat{H}_{\text {eff }}^{\eta, s}$ is defined through the time evolution operator over one period

$\hat{U}(T)=\hat{\mathcal{T}} \exp \left[-i \int_{0}^{T} \hat{H}^{\eta, s}(t) d t\right]=\exp \left[-i \hat{H}_{\mathrm{eff}}^{\eta, s} T\right]$

where $\hat{\mathcal{T}}$ is the time ordering operator. Using perturbation theory and expanding $\hat{U}(T)$ in the limit of large frequencies $\Omega$, we obtain

$$
\begin{aligned}
\hat{H}_{\mathrm{eff}}^{\eta, s}= & \hat{H}_{0}^{\eta, s}+\frac{1}{\hbar \Omega}\left(\left[\hat{H}_{+}^{\eta, s}, \hat{H}_{-}^{\eta, s}\right]\right. \\
& \left.+\left[\hat{H}_{0}^{\eta, s}, \hat{H}_{+}^{\eta, s}\right]-\left[\hat{H}_{0}^{\eta, s}, \hat{H}_{-}^{\eta, s}\right]\right)+O\left(\Omega^{-2}\right),
\end{aligned}
$$

where $\hat{H}_{m}^{\eta, s}=(1 / T) \int_{0}^{T} e^{-i m \Omega t} \hat{H}^{\eta, s}(t) d t$ is the $m$ th Fourier harmonic of the time-periodic Hamiltonian. Notice that Eq. (4) is only valid under the off-resonance condition $\hbar \Omega \gg t_{j}\left(t_{j}=\right.$ $v \hbar / a$ is the hopping parameter between two nearest neighbors with $a$ being the lattice constant) [22,26]. Indeed, for $\Omega \sim t_{j}$ multiple photon absorption and emission processes must be accounted for, which implies that higher order terms in the expansion of $\hat{U}(T)$ should be retained. On the other hand, the condition $\hbar \Omega \gg t_{j}$ implies that the frequency must be large as compared to the bandwidth, which is difficult to reach in practice. In fact, for such large frequencies high energy bands might also contribute to the optical processes. In the present work we focus on the impact of off-resonant light on the low energy bands and assume that direct optical processes involving high energy bands only weakly affect the low energy band structure [27]. Still, by the presence of these high energy processes, the effective power of the incident off-resonant light is reduced.

Using Eq. (1), Eq. (4) yields

$\hat{H}_{\mathrm{eff}}^{\eta, s}=v\left(\eta \hat{\sigma}_{x} \hat{p}_{x}+\hat{\sigma}_{y} \hat{p}_{y}\right)+\left(\Delta \pm \eta \Delta_{\Omega}\right) \hat{\sigma}_{z}-s \eta \lambda \hat{\sigma}_{z}+s \eta \lambda$,

where $\Delta_{\Omega}=e^{2} v^{2} \hbar^{2} A^{2} / \hbar^{3} \Omega^{3}$ is the effective energy term describing the effects of the circularly polarized light, which essentially renormalizes the mass of the Dirac fermions. For right circular polarization the gap is increased in the $K$ valley and reduced in the $K^{\prime}$ valley, whereas for left circular polarization the effect is opposite. After diagonalization we obtain the eigenvalues

$$
E_{\zeta}^{\eta, s}=s \eta \lambda+\zeta \sqrt{(v \hbar k)^{2}+\left(\Delta+\eta \Delta_{\Omega}-s \eta \lambda\right)^{2}}
$$

and the corresponding eigenfunctions

$$
\Psi_{\zeta}^{\eta, s}=\frac{e^{i k_{x} x+i k_{y} y}}{\sqrt{L_{x} L_{y}}}\left(\begin{array}{c}
\cos \gamma_{k}^{\eta, s, \zeta} e^{-i \eta \varphi_{k}} \\
\sin \gamma_{k}^{\eta, s, \zeta}
\end{array}\right) .
$$

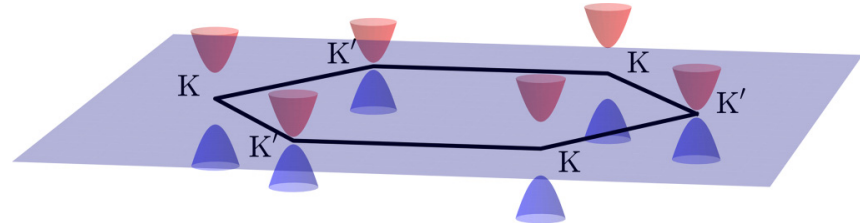

FIG. 1. (Color online) Brillouin zone of monolayer $\mathrm{MoS}_{2}$ and schematic electronic structure in the presence of off-resonant light and absence of intrinsic SOC.

Here $\zeta= \pm 1$ represents the conduction and valence bands, respectively, $\varphi_{k}=\tan ^{-1}\left(k_{y} / k_{x}\right)$ with $k=\sqrt{k_{x}^{2}+k_{y}^{2}}$, and $\tan \gamma_{k}^{\eta, s, \zeta}=\left(E_{\zeta}^{\eta, s}-\Delta-\eta \Delta_{\Omega}\right) / v \hbar k$. The impact of offresonant light on the band structure is illustrated in Figs. 1 and 2 for $\mathrm{MoS}_{2}\left(2 \Delta=1.66 \mathrm{eV}, \lambda=0.0375 \mathrm{eV}, v=0.5 \times 10^{5} \mathrm{~m} / \mathrm{s}\right.$, $a=3.193 \AA$, and $t_{j}=1.10 \mathrm{eV} \mathrm{[5]).} \mathrm{We} \mathrm{set} \hbar \Omega=10 t_{j}$, which corresponds to a gap variation of $\Delta_{\Omega}=0.73 \mathrm{eV}$ for $e v A=$ $2.83 \mathrm{eV}$. This large value of $\hbar \Omega$ ensures that the low energy bands are only affected by virtual emission and absorption processes, while higher energy processes are assumed to only affect the effective power of the incident light (see also Refs. $[22,25,26])$. The energy correction $\Delta_{\Omega}$ can be tuned by varying the amplitude of the electric field or frequency of the light.

Two aspects are worth noticing. First, as mentioned above, the effect of the off-resonant light is to enhance the gap for the $K$ valley and reduce it for the $K^{\prime}$ valley $\left(\Delta_{K}=3.2 \mathrm{eV}\right.$ and $\Delta_{K^{\prime}}=0.06 \mathrm{eV}$ in our example). In this case only one valley (here $\eta=-1$ ) becomes relevant for electronics
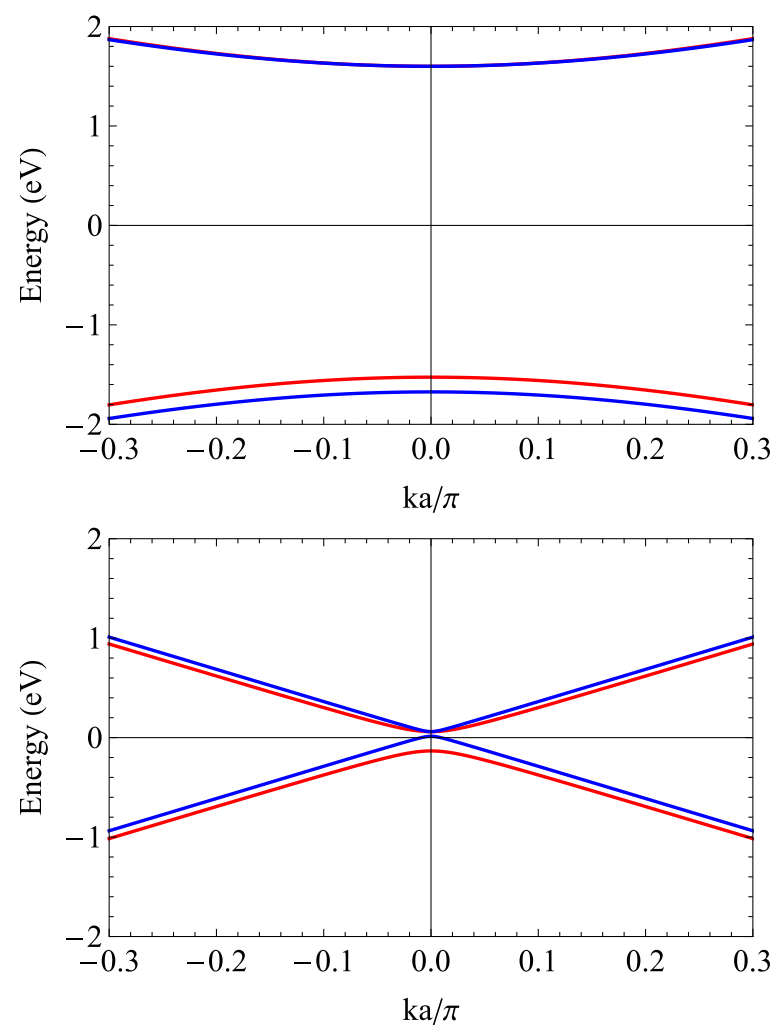

FIG. 2. (Color online) Band structure of monolayer $\mathrm{MoS}_{2}$ in the presence of both off-resonant light and intrinsic SOC for the (top) $K$ and (bottom) $K^{\prime}$ valley. 
purposes, enabling almost $100 \%$ valley polarization of the transport. Second, the SOC-induced splitting is enhanced in the conduction band, while it remains essentially unchanged in the valence band. Indeed, in the absence of off-resonant light the SOC is only active in the valence bands and leaves the conduction bands almost unaffected. The reduction of the band gap in the $K^{\prime}$ valley correspondingly empowers SOC-induced splitting in the conduction bands which become spin polarized. Nevertheless, we stress that due to the fact that the two valence bands $(s= \pm 1)$ are nondegenerate at $k=0$ it is still possible to obtain fully spin-polarized hole transport by tuning the Fermi level, while the conduction band can be at most partially spin polarized. Since the system is fully valley polarized, only the conduction band of the $K^{\prime}$ valley $(\eta=-1)$ contributes to the transport properties discussed in this work.

We calculate the longitudinal conductivity using the Kubo formula and perform a perturbative expansion in terms of the short-range impurity potential within the first Born approximation. We use the Streda [28] version of the Kubo formula [29]

$$
\begin{aligned}
\sigma_{i, j}^{-, s}= & -\frac{e^{2} \hbar}{4 \pi} \int d E \frac{\partial f}{\partial E} \operatorname{Tr}\left[\hat{v}_{i}\left(\hat{G}^{R}-\hat{G}^{A}\right) \hat{v}_{j} \hat{G}^{A}\right. \\
& \left.-\hat{v}_{i} \hat{G}^{R} \hat{v}_{j}\left(\hat{G}^{R}-\hat{G}^{A}\right)\right],
\end{aligned}
$$

where the velocity components $\hat{v}_{i}(i=x, y)$ are given by $\hat{v}_{x}=$ $\eta \hat{\sigma}_{x}$ and $\hat{v}_{y}=\hat{\sigma}_{y}$. The superscript minus represents the $K^{\prime}$ valley, $f$ is the Fermi distribution function, and $\hat{G}^{A / R}$ are the advanced and retarded perturbed Green's functions. The unperturbed retarded Green's function is given by $\hat{G}_{0}^{R}=[E-$ $\hat{H}+i 0]^{-1}$. Using Eq. (5) with $\eta=-1$, we obtain

$$
\begin{aligned}
\hat{G}_{0}^{R}= & \frac{1}{2}\left\{1+\frac{1}{E_{+}^{-, s}+s \lambda}\left[\left(\Delta-\Delta_{\Omega}+s \lambda\right) \hat{\sigma}_{z}\right.\right. \\
& \left.\left.-v \hbar\left(\hat{\sigma}_{x} k_{x}-\hat{\sigma}_{y} k_{y}\right)\right]\right\} \frac{1}{E-E_{+}^{-, s}+i 0} .
\end{aligned}
$$

The perturbed Green's function is given by $\hat{G}^{R}=1$ / $\left[\left(\hat{G}_{0}^{R}\right)^{-1}-\hat{\Sigma}_{R}\right]$, where $\hat{\Sigma}_{R}$ is the retarded self-energy. Considering short range randomly distributed impurities in the first Born approximation, we have

$$
\hat{\Sigma}_{R}=N V_{0}^{2} \int \frac{k d k d \phi}{(2 \pi)^{2}} \hat{G}_{0}^{R} \approx-i N V_{0}^{2} E_{F} / 2 \hbar^{2} v^{2},
$$

where $N$ is the impurity concentration and $V_{0}$ is the impurity potential. We obtain

$$
\begin{aligned}
\hat{G}^{R / A}= & \frac{1}{2}\left\{1+\frac{1}{E_{+}^{-, s}+s \lambda}\left[\left(\Delta-\Delta_{\Omega}+s \lambda\right) \hat{\sigma}_{z}\right.\right. \\
& \left.\left.-v \hbar\left(\hat{\sigma}_{x} k_{x}-\hat{\sigma}_{y} k_{y}\right)\right]\right\} \frac{1}{E \pm i \Gamma-E_{+}^{-, s}},
\end{aligned}
$$

where $\Gamma=-\operatorname{Im} \hat{\Sigma}_{R}$ is the energy broadening due to the finite quasiparticle lifetime $\tau$. Using Eq. (11) in Eq. (8) and addressing the limit of zero temperature with chemical potential $E_{F}$, we arrive at

$$
\sigma_{x x}^{-, s}=\frac{e^{2}}{2 h} \frac{\tau\left(E_{F}+s \lambda\right)}{\hbar}\left\{1-\frac{\left(\Delta-\Delta_{\Omega}+s \lambda\right)^{2}}{\left(E_{F}+s \lambda\right)^{2}}\right\} .
$$

As expected, the conductivity is enhanced under off-resonant light, since the effective band gap is reduced. More interestingly, the gap reduction is accompanied by a spin polarization of the longitudinal electron flow. Indeed, since the mass of the carriers is reduced, the impact of SOC is stronger, leading to a polarization

$$
\begin{aligned}
P & =\left(\sigma_{x x}^{\uparrow}-\sigma_{x x}^{\downarrow}\right) /\left(\sigma_{x x}^{\uparrow}+\sigma_{x x}^{\downarrow}\right) \\
& =\lambda \frac{E_{F}-\left(\Delta-\Delta_{\Omega}\right)}{\left[E_{F}-\left(\Delta-\Delta_{\Omega}\right)\right] E_{F}-2 \lambda^{2}} \underset{\Delta_{\Omega} \rightarrow \Delta}{\longrightarrow} \frac{\lambda E_{F}}{E_{F}^{2}-2 \lambda^{2}} .
\end{aligned}
$$

The intrinsic Hall conductivity due to anomalous trajectories of free electrons under the action of the electric field is expressed in terms of the Berry curvature in $k$ space as $[5,29,30]$

$$
\sigma_{x y}^{\eta, s}=\frac{e^{2}}{\hbar} \int \frac{d^{2} \mathbf{k}}{(2 \pi)^{2}}\left[f_{+}(E)-f_{-}(E)\right] \Omega_{z}^{\eta, s}(\mathbf{k}),
$$

where $f_{ \pm}$denotes the Fermi distributions of the electrons and holes, respectively. From Eqs. (6) and (7) we obtain the Berry curvature as

$$
\begin{aligned}
\Omega_{z}^{\eta, s}(\mathbf{k}) & =i \mathbf{z} \cdot\left\langle\nabla_{\mathbf{k}} \Psi_{\zeta}^{\eta, s}|\times| \nabla_{\mathbf{k}} \Psi_{\zeta}^{\eta, s}\right\rangle \\
& =\frac{-\eta \hbar^{2} v^{2}\left(\Delta+\eta \Delta_{\Omega}-\eta s \lambda\right)}{2\left\{(v \hbar k)^{2}+\left(\Delta+\eta \Delta_{\Omega}-\eta s \lambda\right)^{2}\right\}^{3 / 2}} .
\end{aligned}
$$

Using Eq. (15) in Eq. (14) and performing the integral over $\mathbf{k}$, we obtain the intrinsic Hall conductivity when the Fermi level is in the band gap (indicated by a subscript 0 )

$$
\sigma_{x y, 0}^{\eta, s}=\eta \frac{e^{2}}{2 h} \operatorname{sgn}\left(\left|\Delta+\eta \Delta_{\Omega}\right|-\eta s \lambda\right),
$$

which yields the quantum spin and valley Hall effects. For $\left|\Delta-\Delta_{\Omega}\right|>\lambda$ we have $\sigma_{x y, 0}^{\eta, s}=\eta e^{2} / 2 h$, which results in a vanishing quantum spin Hall effect and finite quantum valley Hall effect $\sigma_{x y, 0}^{v}=\sigma_{x y, 0}^{+, s}-\sigma_{x y, 0}^{-, s} \sim-e^{2} / h$. For $\left|\Delta-\Delta_{\Omega}\right|<$ $\lambda$ we have $\sigma_{x y, 0}^{\eta, s}=-s e^{2} / 2 h$, which results in a vanishing quantum valley Hall effect and finite quantum spin Hall effect $\sigma_{x y, 0}^{s}=\sigma_{x y, 0}^{\eta,+}-\sigma_{x y, 0}^{\eta,-} \sim-e^{2} / h$. Using $\lambda=37.5 \mathrm{meV}$ we obtain for $\left|\Delta-\Delta_{\Omega}\right|=10 \mathrm{meV}$ a value of $\Delta_{\Omega}=0.82 \mathrm{eV}$ for $e v A=3 \mathrm{eV}$, which may be varied by alteration of $\Delta_{\Omega}$ via the intensity of the off-resonant light and is consistent with Fig. 2. These results can be compared with Ref. [6] where the quantum spin Hall effect is zero in the limit of zero off-resonant light, while the quantum valley Hall effect is similar to what we obtain above in Eq. (16), see Eq. (12d) of Ref. [6]. Note that the SOC is stronger in $\mathrm{WS}_{2}(107.5 \mathrm{meV})$ than in $\mathrm{MoS}_{2}$ $(37.5 \mathrm{meV})$ so that the quantum spin Hall effect is easier to detect [5].

When the Fermi level is in the conduction band (indicated by a subscript 1 ), the Hall conductivity is

$$
\sigma_{x y, 1}^{\eta, s}=\eta \frac{e^{2}}{2 h} \frac{\Delta+\eta \Delta_{\Omega}-\eta s \lambda}{E_{F}-\eta s \lambda} .
$$

This anomalous conductivity is similar to that reported in Refs. [5,6] in the limit $\Delta_{\Omega} \rightarrow 0$. However, the valley selectivity introduced by the circularly polarized off-resonant light dramatically changes the situation. Indeed, as mentioned in Refs. [5,6], for monolayer $\mathrm{MoS}_{2}$ in the absence of off-resonant light both valleys contribute equally to the spin and valley Hall 
conductivities. However, in the presence of off-resonant light, since the effective band gap becomes valley dependent, the spin Hall effect is dominated by one valley only $\left(K^{\prime}\right.$ in the present case) so that it is enhanced, while the valley Hall effect is correspondingly reduced. Since the system is fully valley polarized, only the $K^{\prime}$ conduction band contributes, so that the $\operatorname{spin}\left(\sigma_{x y}^{s}=\sigma_{x y, 1}^{-,+}-\sigma_{x y, 1}^{-,-}\right)$and valley $\left(\sigma_{x y}^{v}=\sigma_{x y, 1}^{-,+}+\sigma_{x y, 1}^{-,-}\right)$ Hall conductivities are

$$
\begin{aligned}
& \sigma_{x y}^{s}=-\frac{e^{2}}{h}\left(\frac{\lambda\left(-\Delta+\Delta_{\Omega}+E_{F}\right)}{E_{F}^{2}-\lambda^{2}}\right), \\
& \sigma_{x y}^{v}=-\frac{e^{2}}{h}\left(\frac{\left(\Delta-\Delta_{\Omega}\right) E_{F}-\lambda^{2}}{E_{F}^{2}-\lambda^{2}}\right) .
\end{aligned}
$$

When the gap is quenched $\left(\Delta \rightarrow \Delta_{\Omega}\right)$ the spin Hall effect is simply proportional to the longitudinal polarization, see Eq. (13). The spin and valley Hall conductivities obtained from Eq. (18) can be compared to their counterparts $\sigma_{x y}^{s, 0}$ and $\sigma_{x y}^{v, 0}$ in the absence of light (see, e.g., Eq. (12e) in Ref. [6]) and we obtain

$$
\begin{aligned}
& \sigma_{x y}^{s}=\left(1-\frac{\Delta_{\Omega}}{\Delta-E_{F}}\right) \frac{\sigma_{x y}^{s, 0}}{2}, \\
& \sigma_{x y}^{v}=-\left(1-\frac{\Delta_{\Omega} E_{F}}{\Delta E_{F}-\lambda^{2}}\right) \frac{\sigma_{x y}^{v, 0}}{2},
\end{aligned}
$$

which explicitly reveals the role of the light-induced gap. The extrinsic corrections to the anomalous velocity from side jump and skew scattering have been explicitly calculated in the case of a gapped two-dimensional Dirac Hamiltonian in Ref. [29]. Interestingly, they vanish when the chemical potential approaches the gap. By its large gap $(1.66 \mathrm{eV})$, this is the case for $\mathrm{MoS}_{2}$. Additionally, skew scattering contributions are inversely proportional to the impurity concentration and thus vanish in the limit of strong impurity scattering (see, e.g., Ref. [31]).

The last aspect to be discussed is the enhancement of the orbital magnetic moment. Indeed, in classical electromagnetism, charges moving with a velocity $v$ along a loop of diameter $D$ generate an orbital magnetic moment $\mu_{\mathrm{orb}}=$ Dev/4. Recently, values up to $\mu_{\text {orb }} \approx 26 \mu_{\mathrm{B}}$ have been reported in $5 \mathrm{~nm}$ wide carbon nanotubes [32]. In general, while the orbital contribution to the magnetization is vanishingly small in $3 d$ transition metal ferromagnets, it turns out to become significant in systems involving orbital degrees of freedom such as nanotubes and graphenelike structures [30,33]. The orbital magnetic moment can be related to the Berry curvature through the relation $[30,33]$

$$
\begin{aligned}
\mu_{\mathrm{orb}}^{-, s}(k) & =\frac{e}{\hbar} E_{+}^{-, s}(k) \Omega_{z}^{-, s}(k) \\
& =\frac{e}{\hbar} E_{+}^{-, s}(k) \frac{\hbar^{2} v^{2}\left(\Delta-\Delta_{\Omega}+s \lambda\right)}{2\left\{(v \hbar k)^{2}+\left(\Delta-\Delta_{\Omega}+s \lambda\right)^{2}\right\}^{3 / 2}} .
\end{aligned}
$$

For finite $\Delta$ or $\Delta_{\Omega}$ the orbital magnetic moment has a peak at $k=0$. For zero SOC we obtain for $\left|\Delta-\Delta_{\Omega}\right|=30 \mathrm{meV}$ a single valley orbital magnetic moment of 35 bohrs magnetons. This may be varied by alteration of $\Delta_{\Omega}$ by modifying the intensity of the off-resonant light. The orbital magnetic moment turns out to be inversely proportional to the band gap.

The corresponding orbital magnetization is $[30,33]$

$$
M_{\mathrm{orb}}^{-, s}=\frac{e}{\hbar} \int \frac{d^{2} k}{(2 \pi)^{2}}\left\{\mu_{\mathrm{orb}}^{-, s}(k)+\frac{e}{\hbar}\left[E_{F}-E_{+}^{-, s}(k)\right] \Omega_{z}^{-, s}(k)\right\}
$$

and we calculate analytically

$$
M_{\mathrm{orb}}^{-, s}=\frac{e E_{F}}{2 h}\left(1-\frac{\Delta-\Delta_{\Omega}+s \lambda}{E_{F}+s \lambda}\right) .
$$

Interestingly, this expression has a similar structure as the Hall conductivity, Eq. (17), and can be enhanced by reducing the band gap using off-resonant light. As a reference, for a Fermi energy of $100 \mathrm{meV}$ and a layer thickness of typically $0.6 \mathrm{~nm}$, we would have an orbital magnetization of $0.05 \mathrm{~T}$, which is easily detectable and tunable by varying $\Delta_{\Omega}$. The orbital magnetization can be probed by various experimental techniques, including susceptibility measurements, electron paramagnetic resonance, $\mathrm{x}$-ray magnetic circular dichroism, and neutron diffraction [34-36]. The orbital contribution to the magnetization affects a variety of properties and phenomena such as the nuclear magnetic resonance [37] and electron paramagnetic resonance [38] $g$ tensors, which both are related to the derivative of the orbital magnetization as well as to the magnetic susceptibility, the orbital magnetoelectric response [39-41], and the quantum spin Hall conductivity [42].

We propose to use off-resonant circularly polarized light to enable valley-polarized nanoelectronics in group-VI dichalcogenide monolayers such as $\mathrm{MoS}_{2}$. We theoretically demonstrate that under such illumination the band gaps of the $K$ and $K^{\prime}$ valleys are oppositely tuned, leading to $100 \%$ valley polarization. This phenomenon leads to a number of remarkable effects: (i) Enhancement of the longitudinal conductivity, accompanied by an increase in the spin polarization of the flowing electrons, (ii) enhancement of the intrinsic spin Hall effect, together with a reduction of the intrinsic valley Hall effect, and (iii) enhancement of the orbital magnetic moment and orbital magnetization. Our predictions can be realized experimentally by the setup used in Ref. [43] for studying $\mathrm{WS}_{2}$. The discussed findings expand the horizon of fundamental investigations of the electronic properties of two-dimensional dichalcogenide systems and present promising opportunities to the design of tunable phototransistors [15-20], photothermoelectric devices [44], and related transport devices.

A.M. acknowledges fruitful discussions with Dr. T. Korn. Research reported in this publication was supported by the King Abdullah University of Science and Technology (KAUST).
[1] A. K. Geim and I. V. Grigorieva, Nature (London) 499, 419 (2013).
[2] B. Radisavljevic, A. Radenovic, J. Brivio, V. Giacometti, and A. Kis, Nat. Nanotechnol. 6, 147 (2011). 
[3] K. S. Novoselov, A. K. Geim, S. Morozov, D. Jiang, Y. Zhang, S. Dubonos, I. Grigorieva, and A. A. Firsov, Science 306, 666 (2004).

[4] A. H. Castro Neto, F. Guinea, N. M. R. Peres, K. S. Novoselov, and A. K. Geim, Rev. Mod. Phys. 81, 109 (2009).

[5] D. Xiao, G.-B. Liu, W. Feng, X. Xu, and W. Yao, Phys. Rev. Lett. 108, 196802 (2012).

[6] Z. Li and J. P. Carbotte, Phys. Rev. B 86, 205425 (2012).

[7] K. F. Mak, C. Lee, J. Hone, J. Shan, and T. F. Heinz, Phys. Rev. Lett. 105, 136805 (2010).

[8] A. Splendiani, L. Sun, Y. Zhang, T. Li, J. Kim, C. Y. Chim, G. Galli, and F. Wang, Nano Lett. 10, 1271 (2010).

[9] A. Kuc, N. Zibouche, and T. Heine, Phys. Rev. B 83, 245213 (2011).

[10] T. Cao, G. Wang, W. Han, H. Ye, C. Zhu, J. Shi, Q. Niu, P. Tan, E. Wang, B. Liu, and J. Feng, Nat. Commun. 3, 887 (2012).

[11] K. F. Mak, K. He, J. Shan, and T. F. Heinz, Nat. Nanotechnol. 7, 494 (2012).

[12] H. Zeng, J. Dai, W. Yao, D. Xiao, and X. Cui, Nat. Nanotechnol. 7, 490 (2012).

[13] S. Wu, J. S. Ross, G.-B. Liu, G. Aivazian, A. Jones, Z. Fei, W. Zhu, D. Xiao, W. Yao, D. Cobden, and X. Xu, Nat. Phys. 9, 149 (2013).

[14] A. M. Jones, H. Yu, N. J. Ghimire, S. Wu, G. Aivazian, J. S. Ross, B. Zhao, J. Yan, D. G. Mandrus, D. Xiao, W. Yao, and X. Xu, Nat. Nanotechnol. 8, 634 (2013).

[15] Z. Yin, H. Li, L. Jiang, Y. Shi, Y. Sun, G. Lu, Q. Zhang, X. Chen, and H. Zhang, ACS Nano 6, 74 (2012).

[16] H. S. Lee, S. W. Min, Y. G. Chang, M. K. Park, T. Nam, H. Kim, J. H. Kim, S. Ryu, and S. Im, Nano Lett. 12, 3695 (2012).

[17] W. Choi, M. Y. Cho, A. Konar, J. H. Lee, G.-B. Cha, S. C. Hong, S. Kim, J. Kim, D. Jena, J. Joo, and S. Kim, Adv. Mater. 24, 5832 (2012).

[18] M. Fontana, T. Deppe, A. K. Boyd, M. Rinzan, A. Y. Liu, M. Paranjape, and P. Barbara, Sci. Rep. 3, 1634 (2013).

[19] M. Shanmugam, C. A. Durcan, and B. Yu, Nanoscale 4, 7399 (2012).

[20] O. L. Sanchez, D. Lembke, M. Kayci, A. Radenovic, and A. Kis, Nat. Nanotechnol. 8, 497 (2013).

[21] Y. H. Wang, H. Steinberg, P. J. Herrero, and N. Gedik, Science 342, 453 (2013).

[22] T. Kitagawa, T. Oka, A. Brataas, L. Fu, and E. Demler, Phys. Rev. B 84, 235108 (2011).
[23] T. Kitagawa, M. A. Broome, A. Fedrizzi, M. S. Rudner, E. Berg, I. Kassal, A. A. Guzik, E. Demler, and A. G. White, Nat. Commun. 3, 882 (2012).

[24] M. C. Rechtsman, J. M. Zeuner, Y. Plotnik, Y. Lumer, D. Podolsky, F. Dreisow, S. Nolte, M. Segev, and A. Szameit, Nature (London) 496, 196 (2013).

[25] M. Ezawa, Phys. Rev. Lett. 110, 026603 (2013).

[26] A. Gomez-Leon, P. Delplace, and G. Platero, Phys. Rev. B 89 205408 (2014).

[27] G. Usaj, P. M. Perez-Piskunow, L. E. F. F. Torres, and C. A. Balseiro, arXiv:1406.1711.

[28] P. Streda, J. Phys. C 15, L717 (1982).

[29] N. A. Sinitsyn, J. E. Hill, H. Min, J. Sinova, and A. H. MacDonald, Phys. Rev. Lett. 97, 106804 (2006).

[30] D. Xiao, W. Yao, and Q. Niu, Phys. Rev. Lett. 99, 236809 (2007).

[31] S. Onoda, N. Sugimoto, and N. Nagaosa, Phys. Rev. Lett. 97, 126602 (2006).

[32] E. D. Minot, Y. Yaish, V. Sazonova, and P. L. McEuen, Nature (London) 428, 536 (2004).

[33] T. Thonhauser, Int. J. Mod. Phys. B 25, 1429 (2011).

[34] L. L. Hirsh, Rev. Mod. Phys. 69, 607 (1997).

[35] R. M. White, Quantum Theory of Magnetism (Springer, Berlin, 2007).

[36] E. Beaurepaire, H. Bulou, F. Scheurer, and J.-P. Kappler, eds., Magnetism and Synchrotron Radiation, Springer Proceedings in Physics Vol. 133 (Springer, Berlin, 2010), see also the references therein.

[37] T. Thonhauser, D. Ceresoli, A. A. Mostofi, N. Marzari, R. Resta, and D. Vanderbilt, J. Chem. Phys. 131, 101101 (2009).

[38] D. Ceresoli, U. Gerstmann, A. P. Seitsonen, and F. Mauri, Phys. Rev. B 81, 060409(R) (2010).

[39] A. M. Essin, J. E. Moore, and D. Vanderbilt, Phys. Rev. Lett. 102, 146805 (2009).

[40] A. Malashevich, I. Souza, S. Coh, and D. Vanderbilt, New J. Phys. 12, 053032 (2010).

[41] A. M. Essin, A. M. Turner, J. E. Moore, and D. Vanderbilt, Phys. Rev. B 81, 205104 (2010).

[42] S. Murakami, Phys. Rev. Lett. 97, 236805 (2006).

[43] E. J. Sie, J. W. McIver, Y.-H. Lee, L. Fu, J. Kong, and N. Gedik, arXiv:1407.1825.

[44] M. Buscema, M. Barkelid, V. Zwiller, H. S. J. van der Zant, G. A. Steele, and A. C. Gomez, Nano Lett. 13, 358 (2013). 